\title{
Laparoscopic Insulinoma Enucleation from the Retro-Pancreatic Neck: A Stepwise Approach
}

\author{
Claudius Conrad, MD, PhD, Guillaume Passot, MD, Matthew H. G. Katz, MD, Jason B. Fleming, MD, \\ Michael Kim, MD, Yun Shin Chun, MD, Thomas A. Aloia, MD, Jean-Nicolas Vauthey, MD, and Jeffrey E. Lee, MD \\ Department of Surgical Oncology, Hepato-Pancreato-Biliary Surgery, The University of Texas MD Anderson Cancer \\ Center, Houston, TX
}

\begin{abstract}
Background. Enucleation is the preferred surgical management of small, likely benign, insulinomas. Sparing pancreatic parenchyma and minimizing morbidity are of greater importance in these patients due to their anticipated long survival time. Although a laparoscopic approach is ideal, it can be particularly challenging when the insulinoma is retropancreatic or adjacent to critical vascular structures [i.e., superior mesenteric vein-portal vein confluence (SMV-PV)].

Patient. A 35-year-old woman with neuroglycopenic symptoms and hypoglycemia was diagnosed with hyperinsulinemia. Preoperative CT and EUS-FNA confirmed a 6- $\times$ 9-mm neuroendocrine tumor in the parenchyma of the posterior pancreatic neck along the left lateral aspect of the SMV-PV.

Technique. With the patient in stirrups and arms tucked (French Position), the lesser sac was opened to expose the pancreatic body. A retropancreatic tunnel was created anterior to the SMV-PV and the pancreatic neck encircled with umbilical tape to allow for retraction while
\end{abstract}

Electronic supplementary material The online version of this article (doi:10.1245/s10434-016-5106-6) contains supplementary material, which is available to authorized users.

(C) Society of Surgical Oncology 2016

First Received: 19 November 2015;

Published Online: 9 March 2016

C. Conrad, MD, $\mathrm{PhD}$

e-mail: cconrad1@mdanderson.org minimizing pancreatic manipulation. The insulinoma was definitively identified using intraoperative ultrasound (IOUS). IOUS-guided clip placement facilitated direct identification and permitted safe image-guided enucleation. The enucleation was performed at the parenchymal interface, minimizing the risk of main pancreatic duct injury.

Conclusions. Complete pancreatic neck mobilization and view through the laparoscope along the axis of the PV can facilitate exposure at the challenging location of the retropancreatic neck. Transpancreatic IOUS guidance is crucial to identify and safely enucleate small insulinomas in this location. This totally minimally invasive approach can reduce the morbidity of pancreatic surgery for these patients and permit organ-sparing despite the challenging anatomic location.

DISCLOSURE None of the authors have declared any conflict of interest. 